\title{
Isatuximab, carfilzomib, lenalidomide, and dexamethasone (Isa-KRd) in front-line treatment of high-risk multiple myeloma: interim analysis of the GMMG-CONCEPT trial
}

Lisa B. Leypoldt ${ }^{1}{ }^{1}$, Britta Besemer ${ }^{2}$, Anne Marie Asemissen ${ }^{1}$, Mathias Hänel ${ }^{3}$, Igor Wolfgang Blau ${ }^{4}$, Martin Görner ${ }^{5}$, Yon-Dschun Ko ${ }^{6}$, Hans Christian Reinhardt ${ }^{7}$, Peter Staib ${ }^{8}$, Christoph Mann ${ }^{9}$, Raphael Lutz ${ }^{10}$, Markus Munder ${ }^{11}$, Ullrich Graeven (D ${ }^{12}$, Rudolf Peceny ${ }^{13}$, Hans Salwender $\mathbb{I D}^{14}$, Anna Jauch ${ }^{15}$, Manola Zago ${ }^{16}$, Axel Benner ${ }^{10}{ }^{17}$, Diana Tichy ${ }^{17}$, Carsten Bokemeyer ${ }^{1}$, Hartmut Goldschmidt $\mathbb{D D}^{10}$ and Katja C. Weisel (iD) ${ }^{1 凶}$

(c) The Author(s) 2021

Leukemia (2022) 36:885-888; https://doi.org/10.1038/s41375-021-01431-x

The continuous implementation of novel agents in the treatment of multiple myeloma (MM) has led to significant improvement in survival. Especially the addition of monoclonal antibodies directed against CD38 to standard of care regimens led to significantly deepening responses and improved survival outcomes [1]. However, treatment of high-risk (HR) MM remains challenging with still markedly impaired survival, and risk-adapted treatment concepts are rare $[2,3]$. Even aggressive approaches resulted in two-year median progression-free survival (PFS) rates of approximately 50\% [4]. The GMMG-CONCEPT trial (NCT03104842) investigates the quadruplet regimen isatuximab, carfilzomib, lenalidomide, and dexamethasone (Isa-KRd) in front-line treatment of solely HRMM. Here, we report the interim analysis (IA) focusing on best response during induction and presenting first data on PFS of the first 50 patients.

The IA reports on the first 50 patients included in this phase II, open-label, two-arm, multi-center clinical trial with planned recruitment of 246 patients. Patients were eligible if they had ND symptomatic MM according to international consensus criteria with HR features, defined by the presence of del17p $(\geq 10 \%$ of purified cells) or $\mathrm{t}(4 ; 14)$ or $\mathrm{t}(14 ; 16)$ or $>3$ copies of $1 \mathrm{q} 21$. Furthermore, all patients had to have ISS II or III stage disease [5]. Prior MM-specific treatment was allowed as emergency treatment with a maximum of one cycle of any anti-MM first-line treatment. All patients received ECG and ECHO at screening.

Patients were openly assigned to study arms according to age and transplant eligibility (arm A: patients $\leq 70$ years and eligible for HDT; arm B: patients $>70$ years). Study treatment consisted of six cycles IsaKRd induction, four cycles Isa-KRd consolidation, and Isa-KR maintenance. Transplant-eligible patients underwent HDT with autologous stem cell transplantation (ASCT) after stem cell collection, transplant-ineligible patients received two additional Isa-KRd induction cycles. Primary endpoint of this trial is achievement of minimal residual disease (MRD) negativity measured by next-generation flow after consolidation. Induction treatment with Isa-KRd consisted of six 28-day-cycles with isatuximab $10 \mathrm{mg} / \mathrm{kg}$ of body weight intravenously (i.v.) weekly during the first and on day 1 and 15 of any subsequent cycle, carfilzomib $20 \mathrm{mg} / \mathrm{m}^{2}$ of body surface area i.v. on day 1 and 2 of the first and $36 \mathrm{mg} / \mathrm{m}^{2}$ i.v. on day $8,9,15,16$ of the first and day 1,2 , $8,9,15,16$ of any subsequent cycle, lenalidomide $25 \mathrm{mg}$ orally (p.o.) on day 1-21 of all cycles, and dexamethasone $40 \mathrm{mg}(20 \mathrm{mg}$ for subjects $>75$ years of age) p.o./i.v. on day 1,8,15,22 of all cycles. Prophylactic anticoagulation was obligate and chosen upon the investigator's decision. The population for this IA on overall response rate (ORR) at the end of induction includes the first 50 enrolled patients who received at least one cycle of induction treatment and were eligible for at least one response assessment (46 patients in arm $A$ and 4 patients in arm B). Overall response was determined as the best response until the end of induction including mobilization. Median age was 58 (range: $42-82$ ) years. $56 \%$ of patients showed ISS stage II, 44\% ISS stage III disease. The most common cytogenetic aberration defining HR disease was del17p in $52 \%$ of patients followed by $>3$ copies of $1 q 21$ in $42 \%, t(4 ; 14)$ in $38 \%$ and $t(14 ; 16)$ in

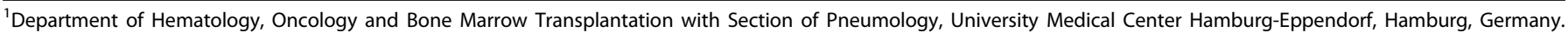
${ }^{2}$ Department of Hematology, Oncology, Immunology, Rheumatology and Pulmonology, University Hospital of Tuebingen, Tuebingen, Germany. ${ }^{3}$ Department of Hematology, Oncology and Bone Marrow Transplantation, Klinikum Chemnitz, Chemnitz, Germany. ${ }^{4}$ Department of Internal Medicine, Charité University Medicine Berlin, Berlin, Germany. ${ }^{5}$ Department of Hematology, Oncology and Palliative Care, Klinikum Bielefeld Mitte, Bielefeld, Germany. ${ }^{6}$ Department of Internal Medicine, Hematology and Oncology, Johanniter Krankenhaus Bonn, Bonn, Germany. ${ }^{7}$ Department of Hematology and Stem Cell Transplantation, University Hospital Essen, University Duisburg-Essen, German Cancer Consortium (DKTK partner site Essen), Essen, Germany. ${ }^{8}$ Department of Hematology and Oncology, St. Antonius Hospital Eschweiler, Eschweiler, Germany. ${ }^{9}$ Department of Hematology, Oncology and Immunology, University Hospital of Gießen and Marburg, Marburg, Germany. ${ }^{10}$ University Hospital Heidelberg, Internal Medicine V and National Center for Tumor Diseases (NCT), Heidelberg, Germany. ${ }^{11}$ Department of Internal Medicine III, University Medical Center Mainz, Mainz, Germany. ${ }^{12}$ Department of Hematology, Oncology and Gastroenterology, Maria Hilf Kliniken, Mönchengladbach, Germany. ${ }^{13}$ Department of Oncology, Hematology and Stem Cell Transplantation, Klinikum Osnabrück, Osnabrück, Germany. ${ }^{14}$ Asklepios Tumorzentrum Hamburg, AK Altona and AK St. Georg, Hamburg, Germany. ${ }^{15}$ Institute of Human Genetics, University of Heidelberg, Heidelberg, Germany. ${ }^{16}$ Center for Clinical Trials, University Hospital of Tuebingen, Tuebingen, Germany. ${ }^{17}$ Division of Biostatistics, German Cancer Research Center (DKFZ) Heidelberg, Heidelberg, Germany. ${ }^{{ }^{\star}}$ email: k.weisel@uke.de
} 
Table 1. Best response during induction.

\begin{tabular}{lccc} 
& $\begin{array}{l}\text { Arm A, } \\
\boldsymbol{N}=\mathbf{4 6}(\%)\end{array}$ & $\begin{array}{l}\text { Arm B, } \\
\boldsymbol{N}=\mathbf{4}(\%)\end{array}$ & $\begin{array}{l}\text { Overall, } \\
\boldsymbol{N}=\mathbf{5 0}(\%)\end{array}$ \\
$\geq$ CR & $23(50)$ & 0 & $23(46)$ \\
SCR & $3(6.5)$ & 0 & $3(6)$ \\
\hline CR & $20(43.5)$ & 0 & $20(40)$ \\
\hline VGPR & $18(39.1)$ & $4(100)$ & $22(44)$ \\
\hline PR & $5(10.9)$ & 0 & $5(10)$ \\
ORR & $46(100)$ & $4(100)$ & $50(100)$ \\
\hline VGPR & $41(89.1)$ & $4(100)$ & $45(90)$ \\
\hline MRD negative & $20 / 31(64.5)$ & $0 / 1(0)$ & $20 / 32(62.5)$ \\
\hline
\end{tabular}

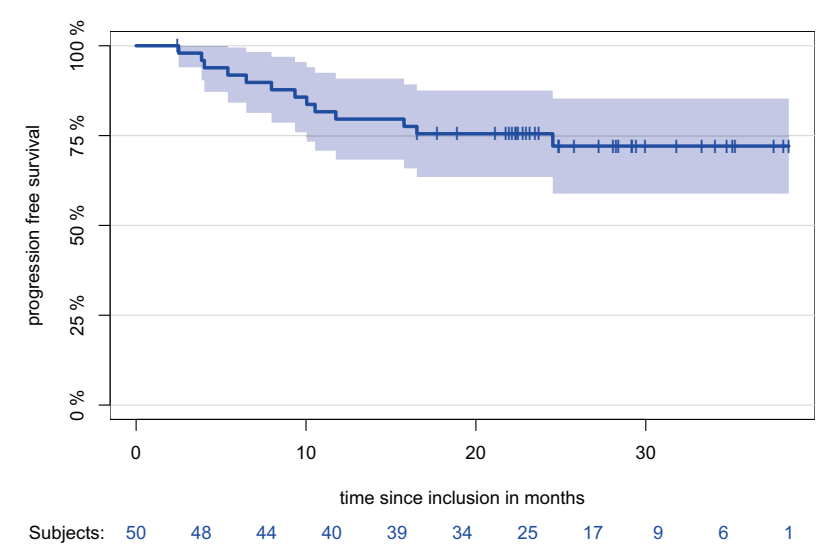

Fig. 1 Progression-free survival (PFS) of the IA population $(\boldsymbol{N}=\mathbf{5 0})$. With a median follow-up of 24.9 months, median PFS was not reached with a median 12-month PFS of $79.6 \%$ (Cl: $68.3 \%$; $90.9 \%$ ) and a median 24-month PFS of $75.5 \%$ (Cl: $63.5 \% ; 87.6 \%)$.

$12 \%$, respectively. 15 patients (30\%) showed $\geq 2$ HR aberrations and $20 \%$ of patients had an elevated LDH.

Forty-four of 50 patients completed induction, seven patients discontinued treatment due to progressive disease $(n=3)$, death $(n$ $=3$ ) or patient's request $(n=1)$. Average dose intensities were $95.7 \%$ for isatuximab, $95.2 \%$ for dexamethasone, $91.6 \%$ for carfilzomib, and $87.9 \%$ for lenalidomide. With regards to the goal of this IA reporting on best response during induction, all patients $(50 / 50$; ORR $=100 \%)$ responded to the induction treatment showing at least a partial response (PR) as best response. $45 / 50$ patients (90\%) showed a VGPR or better, $20 / 50$ patients $(40 \%)$ a complete response (CR) and three patients $(6 \%)$ a stringent complete response (sCR) (Table 1). Of the four patients in treatment arm B, all patients completed induction and achieved VGPR (Table 1).

Median time to first response was 34 days with $95.8 \%$ achieving $\geq P R$ after the first induction cycle. Assessment of MRD during induction was recommended in all patients achieving $\geq V G P R$. In total, 33 patients underwent MRD assessment. Of those, 20 patients were negative, 11 patients positive, two patients were non-assessable. After a median follow-up of 24.9 months, median PFS was not reached with a median 12-month PFS of $79.6 \%$ (Cl: $68.3 \%$; $90.9 \%)$ and a median $24-$ month PFS of $75.5 \%$ (Cl: 63.5\%; 87.6\%) (Fig. 1). Most common adverse events ( $A E$ ) of any grade occurring in $\geq 10 \%$ of patients were neutropenia, lymphopenia, leukopenia, anemia, thrombocytopenia, upper respiratory tract infections, pyrexia, rash, peripheral sensory neuropathy, arterial hypertension, and nasopharyngitis. Most common AEs grade $3 / 4$ occurring in $\geq 10 \%$ of patients were neutropenia, lymphopenia, leukopenia, thrombocytopenia, anemia, infections, and arterial hypertension. Serious adverse events (SAE) of $\geq$ grade 3 occurred in 18 patients, most common SAEs being infectious $(n=5)$ and cardiovascular disorders $(n=5)$. Grade $3 / 4$ cardiac failure was documented in 4 patients, isatuximab-related infusion reactions occured in $32 \%$, all grade 1 or 2 . Death on study during induction phase including mobilization occurred in three patients with two fatal pneumonias and one fatal neutropenic sepsis after stem cell mobilization. Median number of collected CD34 + cells was $6.0 \times$ $10^{6}$ per kg body weight.

Trials for solely HRMM are rare and the proportion of HR patients in first-line phase III trials is generally limited representing around $15-25 \%$ of the total patient population $[6,7]$. Even more, a substantial proportion of ultra HR patients does not enter clinical trials due to aggressiveness of the disease leading to emergency treatment before potential trial inclusion. With one cycle of any myeloma-directed therapy being allowed before enrollment, the GMMG-CONCEPT trial enabled even ultra HR patients including plasma cell leukemia and patients primary non-responding to a first treatment cycle to be included. GMMG-CONCEPT is the first trial investigating the Isa-KRd quadruplet regimen in the treatment of $M M$. This IA focusing on best overall response during induction showed an ORR of $100 \%$ with $90 \%$ of patients achieving $\geq$ VGPR and $46 \%$ showing a CR or SCR and so revealed promising results with no patients primary refractory to the chosen quadruplet combination. MRD analysis during induction was not obligate, however, recommended for all patients achieving at least a VGPR. Of 33 patients tested for MRD at this early time point, 31 were evaluable and of those, 20 were negative for MRD. To address the question whether the achieved early high response rates translate into survival outcome, we conducted a PFS analysis after a median follow-up of 24.9 months demonstrating a two-year PFS rate of $75.5 \%$ with a median PFS not reached. To the best of our knowledge, this is one of the highest described in this unfavorable patient group. Isa-KRd as a quadruplet regimen was tolerable, AEs were clinically manageable and consistent with known toxicities of each individual substance. Reported AEs of interest, especially cardiac toxicities, were within the expected range, rates of peripheral neuropathy were low. Regarding the 3 reported fatal infectious events, with one where a relation to study medication could not be excluded, a careful look on the larger patient population is needed. However, in this difficult-to-treat population, we see a positive risk-benefit analysis outweighing efficacy above toxicity. Currently, there are several trials underway investigating quadruplet regimens in NDMM and even HRMM. In the SWOG 1211 trial for HR patients, addition of the monoclonal antiSLAMF7 antibody elotuzumab to bortezomib, lenalidomide and dexamethasone did not lead to improved outcome [8]. ORR was $83 \%$ with a $2.1 \%$ CR and a $21.3 \%$ VGPR rate in the quadruplet treatment arm, median PFS was 31.47 months [8]. Out of the FORTE trial, Gay and colleagues reported a PFS rate of $62 \%$ after four years in HR patients treated with upfront KRd, ASCT, and KR or R maintenance, however using a broad definition of HR accounting for more than $50 \%$ of the trial population [9]. The UK OPTIMUM HR study investigating quintruplet induction of Dara-CVRD followed by HDT and ASCT in HR patients most recently reported an ORR of $94 \%$ with $a \geq$ VGPR rate of $80 \%$ as the best response during induction [10]. Two recent trials are investigating the anti-CD38-KRd (Dara-KRd) combination not restricted to $\mathrm{HR}$ patients: The single-center MANHATTAN trial reported an ORR of $100 \%$ with a 1 -year-PFS of $98 \%$ in 41 patients [11]. The MASTER trial showed a rate of $90 \%$ $\geq$ VGPR after induction in 70 patients [12]. Taken together, anti-CD38KRd trials particularly underline the high potential in achieving deep responses including MRD-negativity. This might open again the discussion about the future relevance of primary HDT and ASCT.

In summary, our data demonstrate encouraging rates of rapid and deep remissions in HR MM patients with Isa-KRd induction, which may translate into durable responses in this difficult-to-treat patient group and is supported by the first survival data on PFS with a twoyear PFS rate of $75.5 \%$. The trial completed recruitment of the first population of 153 patients early in 2020 and is ongoing with an expansion cohort for a total of 246 patients. Further results will be reported. 


\section{REFERENCES}

1. Kumar SK, Rajkumar V, Kyle RA, van Duin M, Sonneveld $P$, Mateos MV, et al. Multiple myeloma. Nat Rev Dis Prim. 2017;3:17046 https://doi.org/10.1038/ nrdp.2017.46. PMID: 28726797

2. Nandakumar B, Binder M, Dispenzieri A, Kapoor P, Buadi F, Gertz MA, et al. Continued improvement in survival in multiple myeloma (MM) including highrisk patients. JCO. Am Society of Clin Oncol. 2019;37:8039-9.

3. Kazmi SM, Nusrat M, Gunaydin H, Cornelison AM, Shah N, Kebriaei P, et al. Outcomes among high-risk and standard-risk multiple myeloma patients treated with high-dose chemotherapy and autologous hematopoietic stem-cell transplantation. Clin Lymphoma Myeloma Leuk. 2015;15:687-93. https://doi.org/10.1016/j. clml.2015.07.641. Epub 2015 Aug 5. PMID: 26361647; PMCID: PMC4644689

4. Nair B, van Rhee F, Shaughnessy JD Jr, Anaissie E, Szymonifka J, Hoering A, et al. Superior results of Total Therapy 3 (2003-33) in gene expression profiling-defined low-risk multiple myeloma confirmed in subsequent trial 2006-66 with VRD maintenance. Blood 2010;115:4168-73. https://doi.org/10.1182/blood-2009-11255620. Epub 2010 Feb 2. PMID: 20124509; PMCID: PMC2879104

5. Rajkumar SV, Dimopoulos MA, Palumbo A, Blade J, Merlini G, Mateos M-V, et al. International Myeloma Working Group updated criteria for the diagnosis of multiple myeloma. Lancet Oncol. 2014;15:e538-48.

6. Mateos MV, Dimopoulos MA, Cavo M, Suzuki K, Jakubowiak A, Knop S, et al. Daratumumab plus bortezomib, melphalan, and prednisone for untreated myeloma. N Engl J Med. 2018;378:518-28.

7. Moreau P, Attal M, Hulin C, Arnulf B, Belhadj K, Benboubker L, et al. Bortezomib, thalidomide, and dexamethasone with or without daratumumab before and after autologous stem-cell transplantation for newly diagnosed multiple myeloma (CASSIOPEIA): a randomised, open-label, phase 3 study. Lancet 2019;394(Jul):29-38.

8. Usmani SZ, Hoering A, Ailawadhi S, Sexton R, Lipe B, Hita SF, et al. SWOG1211 Trial Investigators. Bortezomib, lenalidomide, and dexamethasone with or without elotuzumab in patients with untreated, high-risk multiple myeloma (SWOG-1211): primary analysis of a randomised, phase 2 trial. Lancet Haematol. 2021;8(Jan):e45-e54. https:// doi.org/10.1016/S2352-3026(20)30354-9. Epub 2020 Dec 22. PMID: 33357482

9. Mina R, Zamagni E, Fazio F, Ledda A, Palmas A, Aquino S, et al. Efficacy of carfilzomib-based induction/consolidation with or without autologous transplant and lenalidomide or carfilzomib-lenalidomide maintenance in high-risk patients in the forte trial, HemaSphere, 2021;5:(S2). EHA 2021 Abstract S182.

10. Kaiser M, Hall A, Walker K, de Tute R, Sadie R, Ingleson E, et al. Depth of response and mrd status in ultra high-risk myeloma and plasma cell leukemia treated with dara-cvrd and augmented autologous transplant: results of the risk-stratified uk optimum/muknine trial, HemaSphere, 2021;5:(S2). EHA 2021 Abstract S181.

11. Landgren $\mathrm{O}$, Hultcrantz $M$, Diamond $B$, Lesokhin AM, Mailankody S, Hassoun $H$, et al. Safety and effectiveness of weekly carfilzomib, lenalidomide, dexamethasone, and daratumumab combination therapy for patients with newly diagnosed multiple myeloma: the MANHATTAN nonrandomized clinical trial. JAMA Oncol. 2021;7(Jun):862-8. https://doi.org/10.1001/jamaoncol.2021.0611. PMID: 33856405; PMCID: PMC8050789.

12. Costa L, Chhabra S, Godby K, et al. Daratumumab, carfilzomib, lenalidomide and dexamethasone (Dara-KRd) induction, autologous transplantation and posttransplant, response-adapted, measurable residual disease (MRD)-based Dara-Krd consolidation in patients with newly diagnosed multiple myeloma (NDMM). Blood 2019;134(Supplement_1):860. https://doi.org/10.1182/blood-2019-123170.

\section{ACKNOWLEDGEMENTS}

We thank the patients who consented to participate in this clinical trial and the clinical research teams at the participating centers. We are grateful to Philippe Moreau, Nikhil Munshi and Lutz Eder of the Data Monitoring Committee for their work in supporting this study. The trial was sponsored by the University Medical Center Hamburg-Eppendorf. Study drug and financial support by Amgen, Celgene | A Bristol Myers Squibb Company and Sanofi.

\section{AUTHOR CONTRIBUTIONS}

$\mathrm{LL}, \mathrm{KW}, \mathrm{AB}, \mathrm{DT}, \mathrm{HG}, \mathrm{AJ}, \mathrm{MZ}$, and CB participated in the conception and design of the study. All authors participated in the analysis and interpretation of data, the writing of the manuscript and the decision to submit for publication. Patient data were collected by $L L$, AMA, BB, MH, IWB, MG, Y-DK, HCR, PS, CM, RL, MM, UG, RP, HS, CB, HG, and KW.

\section{FUNDING}

Open Access funding enabled and organized by Projekt DEAL.

\section{COMPETING INTERESTS}

Dr. Leypoldt reports grants and non-financial support from Celgene | A Bristol Myers Squibb Company, grants and non-financial support from Sanofi, grants and nonfinancial support from Amgen, during the conduct of the study; non-financial support from GSK, non-financial support from Abbvie, outside the submitted work; Dr. Asemissen has nothing to disclose. Dr. Besemer has nothing to disclose. Dr. Hänel reports personal fees from Celgene | A Bristol Myers Squibb Company, personal fees from Novartis, personal fees from Takeda, personal fees from Amgen, during the conduct of the study; Dr. Blau has nothing to disclose. Dr. Görner has nothing to disclose. Dr. Ko has nothing to disclose. Dr. Reinhardt reports personal fees from Abbvie, grants from Gilead, personal fees from Merck, other from CDL Therapeutics $\mathrm{GmbH}$, outside the submitted work; Dr. Staib reports grants, personal fees, nonfinancial support and other from Abbvie, grants, personal fees, non-financial support and other from Amgen, grants, personal fees, non-financial support and other from Celgene | A Bristol Myers Squibb Company, grants, personal fees, non-financial support and other from Janssen-Cilag, grants, personal fees, non-financial support and other from Novartis, grants, personal fees, non-financial support and other from Gilead, grants, personal fees, non-financial support and other from Pfizer, grants, personal fees, non-financial support and other from Roche, outside the submitted work; Dr. Mann has nothing to disclose. Dr. Lutz has nothing to disclose. Dr. Munder reports personal fees and non-financial support from Janssen, personal fees and nonfinancial support from Amgen, grants from Incyte, personal fees and non-financial support from BMS, personal fees from Abbvie, personal fees from Sanofi, personal fees from GSK, personal fees from Takeda, outside the submitted work; Dr. Graeven reports personal fees from Amgen, personal fees and non-financial support from Boehringer Ingelheim, personal fees from Daichi Sankyo, personal fees from Servier, personal fees from Celgene | A Bristol Myers Squibb Company, personal fees from Astra Zeneca, personal fees from Johnson Johnson, non-financial support from Merck, personal fees from MSD, personal fees from BMS, during the conduct of the study; Dr. Peceny reports grants and personal fees from Sanofi Genzyme, grants from Novartis, grants from DRK Blutspendedienst NSTOB, grants from Boehringer Ingelheim Pharma GmbH \& Co KG, grants from Celgene | A Bristol Myers Squibb Company, outside the submitted work; Dr. Salwender reports personal fees from Bristol-Myers Squibb/Celgene, personal fees from Janssen Cilag, personal fees from Glaxo Smith Kline, personal fees from Oncopeptides, personal fees from Takeda, personal fees from Sanofi, personal fees from AbbVie, personal fees from Amgen, outside the submitted work; Dr. Jauch has nothing to disclose. Dr. Zago has nothing to disclose. Axel Benner has nothing to disclose. Dr. Tichy has nothing to disclose. Dr. Bokemeyer reports personal fees from Sanofi Aventis, personal fees from Merck KgA, personal fees from Bristol-Myers Squibb, personal fees from Merck Sharp \& Dohme, personal fees from Lilly Imclone, personal fees from Bayer Healthcare, personal fees from GSO Contract research, personal fees from AOK-Rheinland-Hamburg, personal fees from Novartis, outside the submitted work; Dr. Goldschmidt reports grants, personal fees, non-financial support and other from Amgen, grants, personal fees, non-financial support and other from BMS, grants, personal fees, non-financial support and other from Celgene, grants, personal fees, and other from Chugai, grants, personal fees, non-financial support and other from Janssen, grants, personal fees, non-financial support and other from Sanofi, other from Incyte, other from Molecular Partners, other from Merck Sharp and Dohme (MSD), other from Mundipharma, grants, personal fees, non-financial support and other from Takeda, personal fees and other from Novartis, personal fees from Adaptive Biotechnology, personal fees from GlaxoSmithKline (GSK), outside the submitted work. Dr. Weisel reports grants from AMGEN, grants from Celgene | A Bristol Myers Squibb Company, grants from Sanofi, during the conduct of the study; grants, personal fees and nonfinancial support from Amgen, personal fees and non-financial support from BMS, grants, personal fees and non-financial support from Celgene | A Bristol Myers Squibb Company, personal fees from Adaptive Biotech, grants, personal fees and nonfinancial support from Janssen, personal fees and non-financial support from GSK, personal fees from Karyopharm, grants, personal fees and non-financial support from Sanofi, personal fees and non-financial support from Takeda, personal fees from Oncopeptides, personal fees from Roche, outside the submitted work.

\section{ADDITIONAL INFORMATION}

Correspondence and requests for materials should be addressed to Katja C. Weisel.

Reprints and permission information is available at http://www.nature.com/ reprints

Publisher's note Springer Nature remains neutral with regard to jurisdictional claims in published maps and institutional affiliations. 
(c) Open Access This article is licensed under a Creative Commons

Attribution 4.0 International License, which permits use, sharing, adaptation, distribution and reproduction in any medium or format, as long as you give appropriate credit to the original author(s) and the source, provide a link to the Creative Commons license, and indicate if changes were made. The images or other third party material in this article are included in the article's Creative Commons license, unless indicated otherwise in a credit line to the material. If material is not included in the article's Creative Commons license and your intended use is not permitted by statutory regulation or exceeds the permitted use, you will need to obtain permission directly from the copyright holder. To view a copy of this license, visit http://creativecommons. org/licenses/by/4.0/.

(c) The Author(s) 2021 